\title{
Training of Working Memory Impacts Structural Connectivity
}

\author{
Hikaru Takeuchi, ${ }^{1}$ Atsushi Sekiguchi, ${ }^{2}$ Yasuyuki Taki, ${ }^{1}$ Satoru Yokoyama, ${ }^{2}$ Yukihito Yomogida, ${ }^{2,3}$ Nozomi Komuro, ${ }^{4}$ \\ Tohru Yamanouchi, ${ }^{4}$ Shozo Suzuki, ${ }^{4}$ and Ryuta Kawashima ${ }^{1,2,5}$ \\ ${ }^{1}$ Division of Developmental Cognitive Neuroscience, Institute of Development, Aging and Cancer, Tohoku University, Sendai 980-8575, Japan, ${ }^{2}$ Department \\ of Functional Brain Imaging, Institute of Development, Aging and Cancer, Tohoku University, Sendai 980-8575, Japan, ${ }^{3}$ Japan Society for the Promotion of \\ Science, Tokyo 102-8471, Japan, ${ }^{4}$ Department of Physical Education, Sendai University, Sendai 989-1693, Japan, and ${ }^{5}$ Smart Ageing International Research \\ Center, Institute of Development, Aging and Cancer, Tohoku University, Sendai 980-8675, Japan
}

Working memory is the limited capacity storage system involved in the maintenance and manipulation of information over short periods of time. Individual capacity of working memory is associated with the integrity of white matter in the frontoparietal regions. It is unknown to what extent the integrity of white matter underlying the working memory system is plastic. Using voxel-based analysis (VBA) of fractional anisotropy (FA) measures of fiber tracts, we investigated the effect of working memory training on structural connectivity in an interventional study. The amount of working memory training correlated with increased FA in the white matter regions adjacent to the intraparietal sulcus and the anterior part of the body of the corpus callosum after training. These results showed training-induced plasticity in regions that are thought to be critical in working memory. As changes in myelination lead to FA changes in diffusion tensor imaging, a possible mechanism for the observed FA change is increased myelination after training. Observed structural changes may underlie previously reported improvement of working memory capacity, improvement of other cognitive functions, and altered functional activity following working memory training.

\section{Introduction}

Working memory is the limited capacity storage system involved in the maintenance and manipulation of information over short periods of time (Baddeley, 2003). Individual working memory capacity (WMC) is correlated with a wide range of cognitive functions (Baddeley, 2003). Impaired WMC is associated with neurological and psychiatric disorders (Goldman-Rakic, 1994; Baddeley, 2003), as well as normal aging (Wingfield et al., 1988). Previous neuroimaging studies using diverse imaging methods have investigated neural correlates of working memory and WMC (Baddeley, 2003).

Previous findings have indicated that the integrity of white matter in the frontoparietal regions is associated with WMC and conditions with impaired WMC. Regions in the lateral prefrontal cortex (LPFC) and parietal cortex, especially the inferior parietal lobule (IPL) and intraparietal lobule, are involved with the work-

\footnotetext{
Received Sept. 17, 2009; revised Jan. 9, 2010; accepted Jan. 26, 2010.

This study was supported by Japan Science and Technology Agency (JST)/Research Institute of Science and Technology for Society and JST/Core Research for Evolutional Science and Technology. We thank Y. Yamada for operating the MRI scanner, the participants for their contribution to the study, and all our other colleagues at Institute of Development, Aging and Cancer, Tohoku University, for their support. Part of this work was carried out under the auspices of the Cooperative Research Project Program of the Institute of Development, Aging and Cancer, Tohoku University, where this work was performed. We acknowledge the stimulating discussion at the meeting of the Cooperative Research Project of the Institute of Development, Aging and Cancer, Tohoku University, which partially supported the meeting.

Correspondence should be addressed to Hikaru Takeuchi, Division of Developmental Cognitive Neuroscience, Institute of Development, Aging and Cancer, Tohoku University, 4-1, Seiryo-cho, Aoba-ku, Sendai 980-8575, Japan. E-mail: takehi@idac.tohoku.ac.jp.

D01:10.1523/JNEUROSCI.4611-09.2010

Copyright $\odot 2010$ the authors $\quad 0270-6474 / 10 / 303297-07 \$ 15.00 / 0$
}

ing memory system and are activated during WM performance (Baddeley, 2003; Klingberg, 2006). Further, the structural integrity of white matter in frontoparietal white matter regions is correlated with WMC (Klingberg, 2006). Additionally, several neurological and psychiatric disorders are associated with impaired WMC as well as normal aging characterized by impaired WMC and impaired structural integrity of frontoparietal white matter regions (Moseley, 2002; Kanaan et al., 2005; Chua et al., 2008). So, can WMC and the integrity of white matter structures associated with WMC be increased by any means?

Previous studies have shown working memory training's effects on psychological measures and neural systems. Training on working memory tasks and on other cognitive tasks can improve performance on trained tasks and untrained cognitive tasks (for review, see Perrig et al., 2009) [see also Kawashima et al. (2005) and Uchida and Kawashima (2008)]. Also, altered patterns of brain activity in the frontoparietal cortices during untrained cognitive tasks after working memory training have been demonstrated (Olesen et al., 2004). Nevertheless, to our knowledge, no previously reported study has investigated the effect of working memory training on structural connectivity. Given that structural connectivity underlies WMC, as well as disorders with impaired WMC, both of which are of scientific and clinical interest, it is important to investigate the extent of plasticity in structural connectivity.

In this study, we focused on this uninvestigated issue using intensive adaptive training of working memory. Voxel-based analysis (VBA) of fractional anisotropy (FA) using diffusion tensor imaging (DTI) (Le Bihan, 2003), was applied for the analysis 
of white matter connectivity changes. DTI is used to measure the magnitude and direction of water diffusion (i.e., anisotropy) in brain tissue. FA is a measure of the degree of anisotropy and is thought to be modulated by the degree of myelination, axonal membrane thickness and diameter, and/or the amount of parallel organization of the axons (Basser and Pierpaoli, 1996; Beaulieu, 2002). Thus, FA is interpreted as an indicator of white matter pathway strength, or integrity. We reasoned that the structural connectivity that underlies the working memory network (Klingberg, 2006) would be affected by the training.

\section{Materials and Methods}

\section{Participants}

Eleven healthy, right-handed individuals (eight men, three women) participated. The mean age was 21.7 years (SD, 1.44). All subjects were students at Sendai University. All subjects had normal vision, and none had any history of neurological or psychiatric illness. Handedness was evaluated using the Edinburgh handedness inventory (Oldfield, 1971). Written informed consent was obtained from each subject. This study was approved by the Ethics Committee of Tohoku University. One participant who failed to perform two of our three training tasks was omitted from further analysis.

\section{Procedure}

The working memory training programs were Borland $\mathrm{C}++$ computer programs developed in-house and consisted of three working memory tasks. Participants undertook 2 months of training. Training on each day lasted $\sim 25 \mathrm{~min}$, but the total time depended on the level and time between trials. The working memory training program was provided to each of the participants and was used by the subjects on their personal computers. They were recommended to do the working memory training every day. When this was impossible for any reason (e.g., when they had computer trouble), they were allowed to miss the working memory training. Subjects were also allowed to do the working memory training more than once a day. Responses to each trial were logged in a file on the computer, and every $5 \mathrm{~d}$, subjects were asked to submit the logs by mail so that compliance could be verified. Participants were MRI scanned immediately before and after the 2 month period, and all participants took psychological tests immediately before and after the 2 month period. The experimenter gave feedback about the working memory training to the subjects as necessary.

\section{Training tasks}

Three working memory tasks were presented during each training session: (1) A visuospatial working memory task in which circles were presented one at a time at a rate of $1 / \mathrm{s}$ in a three-by-three grid-like interface. Participants had to remember the location and order of the stimuli. After the presentation of stimuli, participants indicated the location and order of the presented stimuli by clicking on a computer screen with a mouse. (2) "Operation" $N$-back task. Generally in the $N$-back task, participants were asked to memorize a series of stimuli and their temporal order, update the list of recent items, and select the responses that corresponded to the previously observed stimuli, according to the $N$-back rule ( $N$ stimuli ago). In our study, all the $\mathrm{N}$-back tasks were designed to require individuals to push one of several buttons that corresponded to the stimuli that were shown $N$ stimuli ago, continuously during the task period, unlike other $N$-back types, which typically require individuals to push one of two buttons to judge whether the current stimuli and the stimuli that were shown $N$ stimuli ago were the same (Jaeggi et al., 2008). In this "operation" $N$-back task, the stimuli consisted of pairs of numbers, 1 to 4 , shown in a random sequence on the screen (e.g., "1 $1+4$ "). Participants were asked to memorize the sum of the two numbers presented (ranging from 2 to 8 ). The subjects had to push the button on the keyboard that corresponded to $N$ stimuli ago. The level of the task $(N)$ changed based on participants' performance, as described below, and the length of the task changed based on the level of the task. One block of the task consisted of $5 \times N$ stimuli (for example, when $N=3$, the number of the stimuli was 15). (3) "Dual" $N$-back task. In this task, the number stimuli consisted of numbers, 1 to 4 , presented in a random sequence in one of four places on a line. The participants were asked to memorize the location and identity of the stimuli and their temporal order. The subjects then had to push the buttons, 1,2,3, or 4, with their left hand, to indicate the identity of the stimuli $N$ stimuli ago and push the buttons, 5, 6, 7, or 8 , with their right hands to indicate the location of the stimuli $N$ stimuli ago (the locations of buttons 5, 6, 7, and 8 corresponded to the locations of the stimuli; button 5 corresponded to the leftmost stimulus and button 8 corresponded to the right-most stimulus). As was the case with the "operation" $N$-back task, one block of the task consisted of $5 \times N$ stimuli. In all of the three tasks, the level of difficulty was varied by changing the level of the tasks, based on the participants' performance.

When the participants answered the problems correctly, the level of the task increased by one, and when the participants failed to answer the problems correctly three times in a row, the level of the task decreased by one. In the first visuospatial working memory task, the level of difficulty was varied by changing the number of presented stimuli. When participants answered the problem correctly (indicated the correct location of all the stimuli in the presented order), it was regarded as a correct answer. In the "operation" $N$-back task, the level of difficulty was varied by changing the level of $N$. Blocks were regarded as completed correctly when the participants made errors in $<20 \%$ of the trials. In the "dual" $\mathrm{N}$-back task, the level of difficulty was also varied by changing the level of $N$. Blocks were regarded as completed correctly when the participants made errors in $<25 \%$ of the trials for both location and identity identification. Each task of each session ended when subjects accumulated a predetermined number of correct trials.

\section{Image acquisition}

All MRI data acquisition was conducted with a 3-T Philips Intera Achieva scanner. Diffusion-weighted data were acquired using a spin-echo EPI sequence $\left(\mathrm{TR}=10,293 \mathrm{~ms}, \mathrm{TE}=55 \mathrm{~ms}, \mathrm{FOV}=22.4 \mathrm{~cm}, 2 \times 2 \times 2 \mathrm{~mm}^{3}\right.$ voxels, 60 slices, SENSE reduction factor $=2$, number of acquisitions $=$ $1)$. The diffusion weighting was isotropically distributed along 32 directions $\left(b\right.$ value $\left.=1000 \mathrm{~s} / \mathrm{mm}^{2}\right)$. Additionally, a single image with no diffusion weighting $\left(b\right.$ value $\left.=0 \mathrm{~s} / \mathrm{mm}^{2}\right)(b$ value $=0$ image $)$ was acquired. The total scan time was $7 \mathrm{~min}, 17 \mathrm{~s}$. Other than $32 b=1000$ images and one $b$ value $=0$ image, there are acquisitions for phase correction and for signal stabilization and these are not used as reconstructed images.

Then, from the collected images, fractional anisotropy values were calculated. In this study, we chose to set the number of acquisitions to one and instead of increasing the number of acquisitions, the diffusion weighting was isotropically distributed along as many as 32 directions.

\section{Behavioral analysis}

Behavioral data were analyzed using the statistical software, SPSS 16.0. Because the usefulness of working memory training on improving working memory tasks was our primary interest, in the behavioral analysis, differences between the highest performances (highest level of the task that participants answered correctly) in the trained working memory tasks in the first three trained sessions and those in the last three trained sessions were tested using one-tailed paired $t$ tests, with a threshold of $p<$ 0.05 . After that, to show that any test-retest changes as a group were associated with working memory training, simple regression analysis with a threshold of $p<0.05$ was used to test whether there was a positive correlation between the amount of improvement in performance (levels) in the trained working memory tasks and the total amount of training that participants completed. The total amount of training was calculated as follows: $\{$ (number of completed sessions of the visuospatial working memory task) + (number of completed sessions of the "operation" $N$-back task) + (number of completed sessions of the "dual" $N$-back task)\}. Also, for the analysis of the performance of working memory tasks, the performance of trained working memory tasks for each participant in the first and last three sessions was calculated as follows: \{ (highest level of the visuospatial working memory task achieved in the first or last three completed sessions) + (highest level of the "operation" $N$-back task achieved in the first or last three completed sessions) $+2 \times$ (highest level of the "dual" $N$-back task achieved in the first or last three com- 
pleted sessions) (the performance of the dual $N$-back task was multiplied by two because when the level of the dual $N$-back task increased by one, the number of stimuli to be remembered increased by two).

\section{Preprocessing of diffusion imaging data and statistical analysis}

Preprocessing and data analysis were performed using Statistical Parametric Mapping software (SPM5; Wellcome Department of Cognitive Neurology), implemented in Matlab (MathWorks).

Normalization. Before normalization of the FA map, our original skull-stripped $b$ value $=0$ image template was created as follows. (1) The skull of unsmoothed $b$ value $=0$ images from the pretraining scans of all subjects in this study were stripped by masking the images by intensity thresholding of the spatially smoothed (using $8 \mathrm{~mm}$ FWHM) $b$ value $=0$ image of each participant. (2)Then, using the affine and nonlinear spatial normalization algorithm, this skull-stripped unsmoothed $b$ value $=0$ image was spatially normalized to the skull-stripped T2 template of SPM5, which was made by masking the images by intensity thresholding of the original SPM5's T2 template. Templates of SPM are based on averages taken from 152 brains from the Montreal Neurological Institute database. By applying the parameters derived from the normalization of the skull-stripped unsmoothed $b$ value $=0$ image, the skull-unstripped $b$ value $=0$ image was also normalized. (3) This normalized skullunstripped $b$ value $=0$ image from the pretraining scan was then smoothed using a Gaussian kernel of $8 \mathrm{~mm}$ FWHM, and then averaged over all 10 subjects. We used a normalizing procedure which follows the creation of a study-specific template. This is because the demographics of our subject population might differ from those used to generate the existing template and because each scanner introduces specific nonuniformities in image intensity and inhomogeneities in the $b$ value $=0$ field. So use of the study-specific template is thought to optimize the normalizing procedure. The procedure for making the study-specific template was taken in the optimized VBM method (Good et al., 2001) (see also Gaser, 2006). Studies have shown the usefulness of making a study-specific template for the normalizing procedure from the normal subject group such as those described above, in VBM and fMRI studies (Hsu et al., 2007; Shen et al., 2007).

The pretraining and posttraining images can be very accurately matched using within-subject registration methods (Collignon et al., 1995), and this would lead to more accurate results. So, the skullunstripped $b$ value $=0$ image from the posttraining scan, which kept alignment with the FA map image from the posttraining scan, was coregistered to the skull-unstripped $b$ value $=0$ image from the pretraining scan using the within-subject registration method. Using the affine and nonlinear spatial normalization algorithm, the skull-unstripped $b$ value $=0$ image from the pretraining scan of each participant was normalized to our original skull-unstripped $b$ value $=0$ image template. By applying the parameters derived from the normalization of the skullunstripped $b$ value $=0$ image from the pretraining scan of each participant, the FA map images from both pretraining and posttraining scans of each participant were spatially normalized to give images with $2 \times 2 \times 2$ $\mathrm{mm}^{3}$ voxels.

Smoothing. Then, the normalized FA map image was spatially smoothed using a Gaussian kernel of $10 \mathrm{~mm}$ FWHM. The smoothing step confers a few important benefits. First, it improves the signal-tonoise ratio which enhances detectability of the genuine effects of interest. Second, it helps to ensure that the assumptions underlying the theory of Gaussian random fields (i.e., Gaussian distribution and homoscedasticity of residuals to the linear model) are met so that the correction for multiple comparisons can be correctly performed. Third, it mitigates the effect of imperfections in the spatial normalization of the constituent images in the dataset (Ashburner and Friston, 2001; Bookstein, 2001). We chose $10 \mathrm{~mm}$ as a smoothing parameter for the two reasons cited below. First, Jones et al. (2005) investigated the effect of smoothing parameter in VBA of DT-MRI data, and in their study, neither small smoothing parameters such as those $<9 \mathrm{~mm}$ nor large smoothing parameter such as those $>14 \mathrm{~mm}$ were appropriate or sensitive enough to find the effects of interest. Second, their study also showed that a smaller smoothing parameter resulted in an increasing number of voxels that had

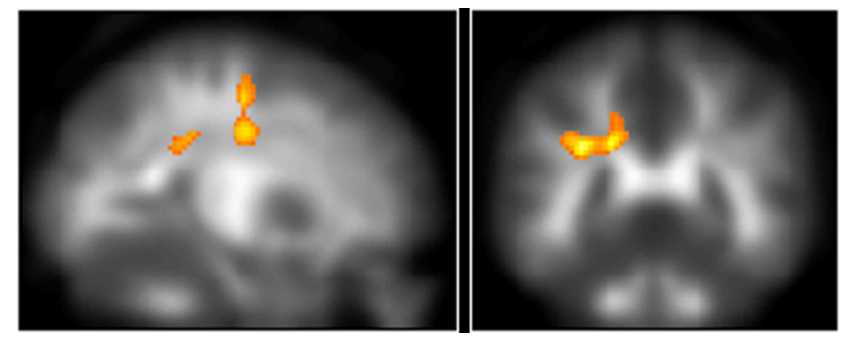

Figure 1. The cluster adjacent to the inferior parietal sulcus, which increased FA significantly after training, together with the significant cluster in the frontal lobe adjacent to the border between the frontal lobe and the parietal lobe ( $p<0.05$, after correction for multiple comparisons at cluster size, with a voxel-level cluster-determining threshold of $p<0.005$ uncorrected).

non-normally distributed residuals, thus making valid statistical inferences with a parametric approach problematic in these areas.

The first group-level analysis. The resulting maps representing the FA of each participant in the pre- and post-MRI experiments were then forwarded to the group paired- $t$ analysis described below. In the wholebrain analysis, using a paired- $t$ test, we investigated regions that showed increased FA following working memory training. In this computation, we included only voxels that showed FA values $>0.2$. This cutoff value was used because FA is susceptible to error arising from partial volumes (Pfefferbaum and Sullivan, 2003) and because with this FA cutoff value, we can dissociate white matter structure from other tissues (Salat et al., 2005). This cutoff value resulted in a search volume of 106,908 voxels.

The second group-level analysis. Finally, the signal change in FA between preintervention and postintervention images was computed at each voxel for each participant. The resulting maps representing the FA change between the pretraining and posttraining MRI experiments were then forwarded to the group regression analysis described below.

Using regression analyses, the effect of working memory training on structural changes was estimated by testing the relationship between the amounts of FA change between pretraining and posttraining measures at each voxel and the amount of working memory training that each subject completed. Such tests were performed because participants were university students who studied and played sports in their daily lives, possibly affecting brain structure (Draganski et al., 2006; Jäncke et al., 2009), and also because there may simply be time-lapse changes in brain structure (Barnea-Goraly et al., 2005). We did not perform a correlation analysis using working memory performance improvement as a covariate because in our study, the improvement of performance seems to be rather affected by participants' initial performance (see Results for details). Here, this voxel-based correlation analysis was performed among regions that were identified as showing significant changes in the paired- $t$ analysis ("inclusive" masking). In this inclusive masking procedure, a mask comprised of areas that were identified as showing significant changes in the paired- $t$ analysis (Fig. 1) ( $p<0.05$, after correction for multiple comparisons at cluster size, with a voxel-level cluster-determining threshold of $p<0.005$ uncorrected; see Statistics section below) was applied to the results of voxel-based correlation analysis. In other words, we restricted the results of the voxel-based correlation analysis to voxels that showed significant FA increase in the paired- $t$ analysis. This resulted in a search volume of 1160 voxels in the voxel-based correlation analysis. This "inclusive" masking reveals voxels that show two or more experimental effects (in this case, FA increase after training and correlation between the FA increase and amount of training), allowing characterization of brain areas sensitive to multiple experimental factors.

In addition to this voxel-based correlation analysis, we performed another correlation analysis. Here, simple regression analyses were performed for the amount of training and the mean FA changes in the clusters identified as significant in the paired- $t$ analysis (See Discussion for the reasons why we performed both of the correlation analyses described above). 
Table 1. Performance and amount of training for the three training tasks (mean \pm SD)

\begin{tabular}{llll}
\hline & $\begin{array}{l}\text { Highest level achieved } \\
\text { in the first three } \\
\text { sessions }\end{array}$ & $\begin{array}{l}\text { Highest level achieved } \\
\text { in the last three } \\
\text { sessions }\end{array}$ & $\begin{array}{l}\text { Number of completed } \\
\text { training sessions }^{a}\end{array}$ \\
\hline $\begin{array}{l}\text { Visuospatial WM task } \\
\text { Operation N-back }\end{array}$ & $3.00 \pm 0.89 \pm 0.75$ & $8.90 \pm 0.94$ & $40.5 \pm 12.4$ \\
Dual N-back & $2.50 \pm 0.50$ & $3.30 \pm 1.10$ & $39.1 \pm 12.7$ \\
\hline
\end{tabular}

${ }^{a}$ Note unless there was some reason for subjects to forgo completion of a task, the number of training sessions for the three tasks would have been the same because subjects were required to do all the tasks. In addition, since subjects were required to do the three training tasks in the same order, the number of training sessions in the first training task (the visuospatial WM task) was the highest.

Statistics. Regions with significance were inferred using cluster-level statistics (Friston et al., 1996). In this procedure, the null hypothesis was rejected when the clusters had a large spatial extent. The distribution of cluster sizes can be found by parametric methods based on the theory of Gaussian random fields, which accounts for image volume, smoothness, and the cluster defining threshold. In cluster level, inference is done at the cluster size; that is the probability that any cluster larger than the critical cluster size is controlled. Only clusters with a $p<0.05$, after correction for multiple comparisons at cluster size, with a voxel-level clusterdetermining threshold of $p<0.005$ uncorrected, were considered statistically significant in this analysis. The voxel-level cluster-determining threshold for cluster level analysis was chosen in this study because in the cluster level test of random field theory, a higher voxel-level clusterdetermining threshold such as $p<0.001$ resulted in a slightly anticonservative test under lower degrees of freedom, as in our experiment (Hayasaka and Nichols, 2003) and, as another study suggested that too low a voxel-level cluster-determining threshold, such as $T$-score $<2$ (which corresponds to approximately $p<0.05$ under the degree of freedom of a typical MRI group-level analysis), also resulted in an anticonservative test (Poline et al., 1997). In the voxel-based correlation analysis, for areas with a strong a priori hypothesis, namely, the white matter regions adjacent to the dorsolateral prefrontal cortex (DLPFC), the anterior part of the body of the corpus callosum, and the genu of the corpus callosum, which connects the bilateral DLPFC (Barbas and Pandya, 1984), an uncorrected $p$ value of $\leq 0.005$, which corresponded to $p<0.07$ corrected for false discovery rate (FDR) (Genovese et al., 2002) in this analysis, was considered significant.

\section{Results}

\section{Behavioral performance}

The highest performance in the trained working memory tasks among the last three training sessions was significantly increased compared to that of the first three training sessions (paired $t$ test, $t=5.01 ; \mathrm{df}=9, p<0.001$; see Materials and Methods for details on the calculation of the performance of trained working memory tasks). This increase in the performance of trained working memory tasks in each subject was significantly and positively correlated with the amount of training that each subject had completed (linear regression, $t=3.41 ; r=0.76$; $\mathrm{df}=8, p=$ $0.009)$. Performance changes and the number of training sessions for each task are provided in Table 1. Furthermore, there was a statistical tendency of negative correlation between the increase in the performance of trained working memory tasks in each subject and each participant's highest performance of trained working memory tasks in the first three training sessions (linear regression, $t=-2.05 ; r=-0.59 ; \mathrm{df}=8, p=0.075$ ), suggesting the inappropriateness of using increase in the performance of trained working memory tasks as a covariate to show the effect of working memory training on structural connectivity in this study. There was no relationship between each participant's total amount of working memory training and each participant's highest performance of trained working memory tasks in the first three training sessions (linear regression, $t=-0.37 ; r=-0.13$; $\mathrm{df}=8, p=0.72$ ).
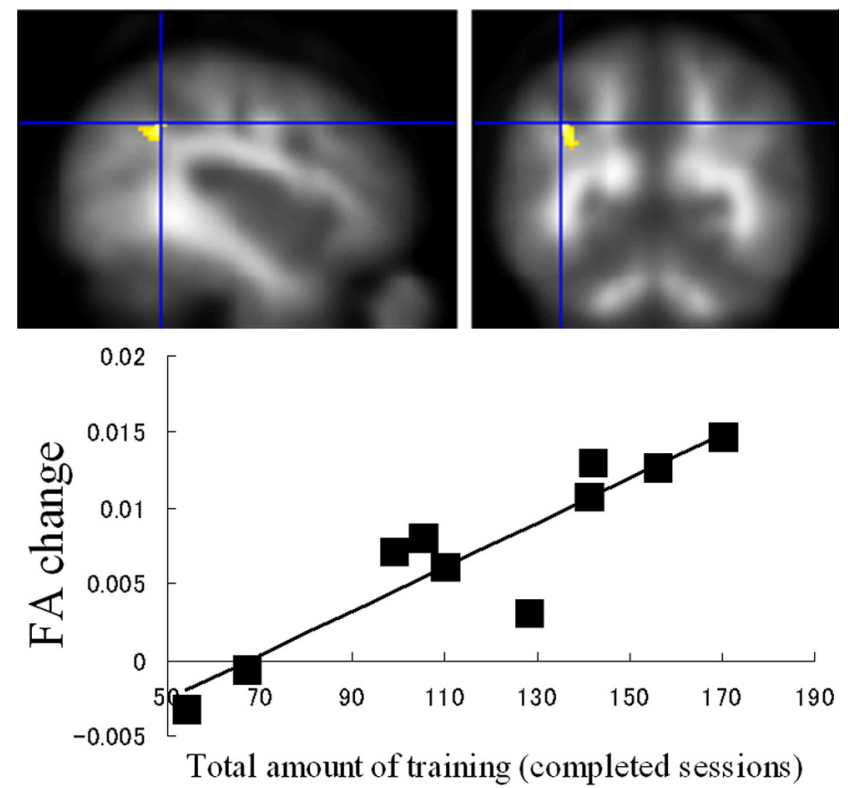

Figure 2. Working memory training and white matter structure changes. Increased FA after training in the white matter region adjacent to the intraparietal sulcus showed a significant correlation with amount of working memory training, overlaid on the mean smoothed FA image from all participants ( $p<0.05$, after correction for multiple comparisons at cluster size, with a voxel-level cluster-determining threshold of $p<0.005$ uncorrected). In the graph below, the effect of working memory training at the peak voxel in this cluster was highlighted.

\section{VBA of diffusion tensor imaging data}

\section{FA change following the training}

Initially in the VBA, we used a paired- $t$ test to assess whether there were group changes in FA after training. Then among the regions that showed group changes in FA after training, we performed a simple linear regression analysis. In this analysis, we tested for associations between the changes in FA from pretraining to posttraining measures and each subjects' total amount of training during the training period to see whether the change in FA was associated with working memory training. A significant increase in FA was found in an anatomical cluster in a white matter region adjacent to the inferior parietal sulcus (IPS) $(x, y, z=-8,-24$, 40 ; paired $t$ test, $t=7.96 ; \mathrm{df}=9, p<0.001$, corrected for multiple comparisons at the cluster level, with a cluster-determining threshold of $p<0.005$, uncorrected) (Fig. 1), in an anatomical cluster that spreads around the body of the corpus callosum and adjacent frontal lobe white matter regions $(x, y, z=-4,24,26$; paired $t$ test, $t=6.18 ; \mathrm{df}=9, p=0.050$, corrected for cluster size) and in an anatomical cluster in a frontal lobe white matter region near the border between the frontal and parietal lobe $(x, y, z=$ $-16,-4,38$; paired $t$ test, $t=5.74$; df $=9, p=0.007$, corrected for cluster size). There were no areas that showed significantly reduced FA following the training.

\section{Effect of training on FA change}

There was a significant positive correlation between the increase in FA after training and the amount of training in a white matter region adjacent to the IPS in the first anatomical cluster (IPS) that was identified in the paired- $t$ analysis $(x, y, z=-40,-50,38$; linear regression, $t=6.37 ; \mathrm{df}=8, p=0.006$, corrected for cluster size) (Fig. 2). There was a significant positive correlation between the increase in FA after training, and amount of training, in a white matter region adjacent to the body of the corpus callosum in the second anatomical cluster that was identified in the first analysis $(x, y, z=-14,14,30$; linear regression, $t=3.55$; $\mathrm{df}=8$, 

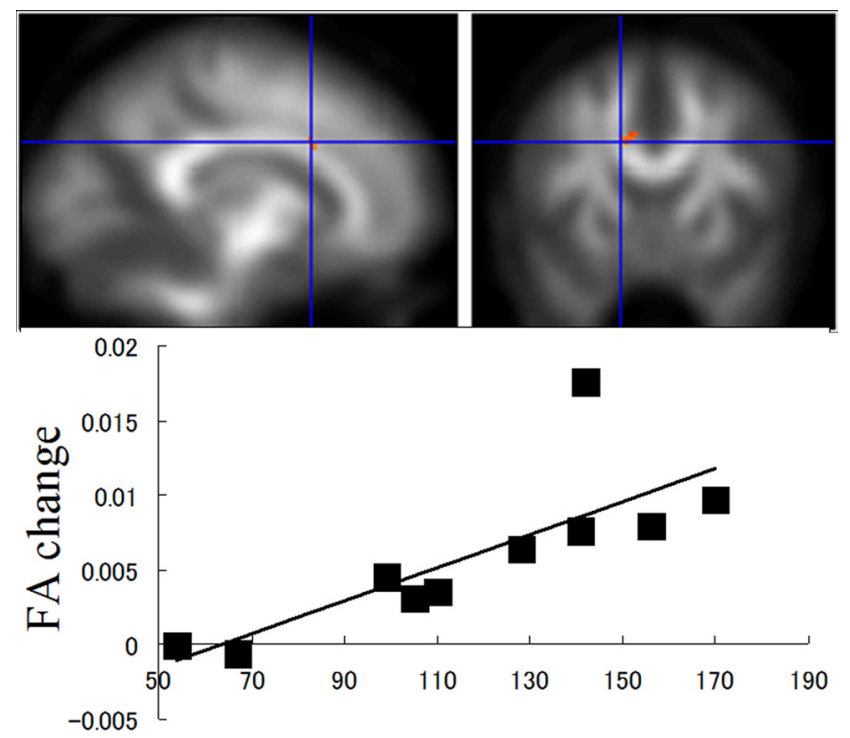

Total amount of training (completed sessions)

Figure 3. Working memory training and white matter structure changes. Increased FA after training in the white matter region adjacent to the anterior part of the body of corpus callosum showed a significant correlation with the amount of working memory training, overlaid on the mean smoothed FA image from all participants ( $p<0.005$ uncorrected, which corresponds to $p<0.07$, corrected for false discovery rate, for the regions with a high a priori hypothesis). In this figure, the identified regions are shown with a threshold of $p<0.05$, uncorrected for visualization purposes. In the graph below, the effect of working memory training at the peak voxel in this cluster was highlighted.

$p=0.004$ uncorrected, which corresponds to $p=0.06$, corrected for false discovery rate, for the regions with a high a priori hypothesis) (Fig. 3). No significant negative correlations were observed in the clusters that were identified in the paired- $t$ analysis. In addition to the voxel-based correlation analysis between the amount of training and FA change, a correlation analysis was performed between the amount of training and mean FA changes in the clusters that showed a significant FA increase in the paired- $t$ analysis. Mean FA change in the cluster in the white matter region adjacent to the IPS showed significant correlation with amount of training (linear regression, $t=3.77 ; r=0.80$; $\mathrm{df}=8, p=0.005$ ), while mean FA changes did not show significant correlation in the cluster within two anatomical clusters; the first of which spreads around the body of the corpus callosum and the adjacent frontal lobe white matter regions (linear regression, $t=1.35 ; r=0.43 ; \mathrm{df}=8, p=0.214)$, and the second of which is in a frontal lobe white matter region near the border between the frontal and parietal lobe (linear regression, $t=-0.06$; $r=-0.02 ; \mathrm{df}=8, p=0.953)$.

\section{Discussion}

The present study revealed the effect of working memory training on structural connectivity. A novel finding is that working memory training increased the structural integrity of the white matter region in the parietal regions and the white matter region adjacent to the body of the corpus callosum, both of which are thought to be important in working memory. It is unlikely that these findings can be explained by the effect of normal development or other factors because increased structural integrity was associated with the amount of working memory training.

Working memory training was associated with FA increases in the white matter region close to the IPS, which connects working memory nodes and may underlie the increased WMC after train- ing. Several studies have explored the interconnections between the intraparietal and frontal areas (Goldman-Rakic, 1988). For example, one previous study (Selemon and Goldman-Rakic, 1988) showed that IPL regions close to the IPS were connected to the prefrontal cortex, and projections from this region and the dorsolateral prefrontal cortex have common projections to at least 15 common cortical targets, suggesting that this region is part of the larger neural circuitry. On the other hand, the inferior and intraparietal cortexes are activated in response to a wide range of working memory demands (Klingberg, 2006). A metaanalysis of imaging studies of working memory showed that a region around the IPS was involved with executive functions in working memory (Wager and Smith, 2003), suggesting that this region plays a key role in brain circuitry that is relevant to working memory. Consistent with this, activity in the intraparietal region, together with the superior frontal sulcus, correlated with working memory capacity (Olesen et al., 2003). Furthermore, values of FA in regions in the frontoparietal network were positively correlated with working memory performance and working-memory-related functional activity (Olesen et al., 2003). These correlations in the previous study are consistent with (1) a training-related increase in FA in this study, (2) a training-related increase in performance of the working memory task in this and a previous study (Olesen et al., 2004) (for review, see Perrig et al., 2009), and (3) a training-related increase in functional activity (Olesen et al., 2004) in a previous study. Thus, the increase in FA in the white matter region adjacent to the IPS may underlie the increased performance and activity change during working memory tasks after training (Olesen et al., 2004).

Working memory training was associated with increases of white matter structural integrity in the white matter adjacent to the anterior part of the body of the corpus callosum, which connects the bilateral DLPFCs, the key nodes of working memory, suggesting that working memory training enhances WMC through increased interhemispheric information transfer between the bilateral DLPFCs. The DLPFC is the key node of the working memory system and this region is also one of the regions that are thought to be engaged in the central executive function in working memory (Baddeley, 2003). The anterior part of the body of the corpus callosum connects the bilateral DLPFC (Barbas and Pandya, 1984) and is thus considered to be involved with the bihemispheric transfer of information that involves the DLPFC. Thus, increased white matter structural integrity in this corpus callosum region, which was caused by working memory training, may lead to increased interhemispheric transfer between the key nodes of working memory and enhance WMC. Nevertheless, compared with the association between FA change adjacent to the IPS and working memory training, the association between FA change in the anterior part of the brain and training was minimal. One possible cause of this may be the modality of the training tasks in this study (all the training tasks in this study used visual stimuli). A previous neuroimaging study (Crottaz-Herbette et al., 2004) investigated the difference between the neural correlate of visual verbal working memory and auditory verbal working memory. The left posterior parietal cortex, primarily along the IPS showed greater responses during visual verbal working memory whereas the left DLPFC showed greater responses during auditory verbal working memory. Perhaps these kinds of the differences are also observed between the effect of visual verbal working memory training and the effect of auditory verbal working memory training. This will be an interesting research subject to investigate in the future. 
We suggest that increased myelination, caused by neural activity in fiber tracts during working memory training, is one possible mechanism underlying the observed FA increases after working memory training. In the mouse, induction of myelination by neural activity has been demonstrated both in vivo and in vitro (Demerens et al., 1996), and the myelination process was affected by and could be manipulated from the outside within a week (Koenig et al., 1995), as well as being affected by environmental influences (Juraska and Kopcik, 1988). It has been well established that myelination continues even into adulthood (Yakovlev and Lecours, 1967; Benes et al., 1994; Nuñez et al., 2000). Furthermore, preliminary research has suggested that myelination remains sensitive to experience, even in adulthood (Markham and Greenough, 2004). Additionally, human studies using DTI showed practice-induced white matter plasticity, even in adulthood (Bengtsson et al., 2005). Our results fit with the idea that skill learning, and experiences, which clearly continue throughout life, are accompanied by structural changes, including myelination (Koester, 1985).

The increase in FA following training may be associated with the enhanced effectiveness of communication between neural circuits, which, in turn, leads to enhanced performance of WM tasks. This is for the following reasons. An action potential spreads faster along myelinated axons than unmyelinated ones (Bloom et al., 1988). In addition, the velocity of conduction of the action potential spread increases with increased myelin thickness in myelinated neuron fibers (Waxman, 1980). Faster conduction velocity can facilitate information flow not only by speeding it up but also by allowing for precise temporal coding of highfrequency bursts of neuronal activity (McDonald and Sears, 1970; Swadlow, 1985; Shrager, 1993). Furthermore, integrity in the timing of sequential events in neuronal circuits could lead to more effective cognitive performance (Peters, 2002).

To show that FA changes in the clusters that showed significant FA increase after training were correlated with amount of working memory training, we performed two correlation analyses: one with FA change at each voxel (VBA) and the other with mean FA change in the significant clusters that showed significant FA increase after training. The reason we performed a voxelbased correlation analysis in addition to the correlation analysis with mean FA changes in the clusters, was because averaging the strength of the signals in clusters with significant FA increase after training, whose voxels can include noise or other effects, is not the best way to look at the effects of training in the region of a significant cluster. Cluster level inference does not statistically guarantee that every single voxel in the significant cluster shows the effect of the contrast (Friston et al., 1996). Rather, the significant cluster in the cluster level analysis can include voxels that showed a statistical tendency because of the noise or, in our study's case, that showed a statistical tendency because of other effects (such as time-lapse change).

There are a few limitations to this study. One is that the subjects' characteristics might affect the results of morphological data, because different subject characteristics (e.g., age) affect differential time-lapse changes in brain structure (Shaw et al., 2006). Other limitations are the limited number of participants and number of $b$ value $=0$ images in our protocol. In our study, because of our MRI scanner's limitation, only a single $b$ value $=0$ image (compared with $32 b$ value $=1000$ images) has been acquired, even though previous studies (Jones et al., 1999, 2002) recommended that $10 \%$ of total images acquired be with lower diffusion weighting for precision in estimates of diffusivity. The low statistical power caused by these factors might prevent us from finding significant training effects on FA in other regions that are important to working memory, such as the right prefrontal cortex. Also, because of our within-subject registration between the pretraining and posttraining images, the posttraining images undergo one interpolation operation more than the pretraining images. This may be a potential pitfall in finding differences between pretraining and posttraining data, although individual differences and their correlation with amount of training cannot be explained by this problem. Finally, although the correlation between FA change and amount of training are impressive in and of themselves, it is still possible, (though unlikely), that FA could have changed over the time period for other reasons.

In summary, the present results show the effects of working memory training on white matter structures that are important in working memory. White matter brain structure underlies cognitive abilities (Olesen et al., 2003), as well as several neurological and psychiatric disorders and normal aging (Moseley, 2002; Kanaan et al., 2005; Chua et al., 2008). Thus, the idea that a brain structure can be changed through short-term cognitive training may provide the basis for new insights into neural plasticity, and may have clinical applications (Klingberg et al., 2002).

\section{References}

Ashburner J, Friston KJ (2001) Why voxel-based morphometry should be used. Neuroimage 14:1238-1243.

Baddeley A (2003) Working memory: looking back and looking forward. Nat Rev Neurosci 4:829-839.

Barbas H, Pandya DN (1984) Topography of commissural fibers of the prefrontal cortex in the rhesus monkey. Exp Brain Res 55:187-191.

Barnea-Goraly N, Menon V, Eckert M, Tamm L, Bammer R, Karchemskiy A, Dant CC, Reiss AL (2005) White matter development during childhood and adolescence: a cross-sectional diffusion tensor imaging study. Cereb Cortex 15:1848-1854.

Basser PJ, Pierpaoli C (1996) Microstructural and physiological features of tissues elucidated by quantitative-diffusion-tensor MRI. J Magn Reson B 111:209-219.

Beaulieu C (2002) The basis of anisotropic water diffusion in the nervous system-a technical review. NMR Biomed 15:435-455.

Benes FM, Turtle M, Khan Y, Farol P (1994) Myelination of a key relay zone in the hippocampal formation occurs in the human brain during childhood, adolescence, and adulthood. Arch Gen Psychiatry 51:477-484.

Bengtsson SL, Nagy Z, Skare S, Forsman L, Forssberg H, Ullén F (2005) Extensive piano practicing has regionally specific effects on white matter development. Nat Neurosci 8:1148-1150.

Bloom FE, Lazerson A, Hofstadter L (1988) Brain, mind, and behavior. New York: Freeman.

Bookstein FL (2001) "Voxel-based morphometry" should not be used with imperfectly registered images. Neuroimage 14:1454-1462.

Chua TC, Wen W, Slavin MJ, Sachdev PS (2008) Diffusion tensor imaging in mild cognitive impairment and Alzheimer's disease: a review. Curr Opin Neurol 21:83-92.

Collignon A, Maes F, Delaere D, Vandermeulen D, Suetens P, Marchal G (1995) Automated multi-modality image registration based on information theory. In: The proceedings of information processing in medical imaging (Bizais Y, Barillot C, DiPaola R, eds). Dordrecht, The Netherlands: Kluwer Academic.

Crottaz-Herbette S, Anagnoson RT, Menon V (2004) Modality effects in verbal working memory: differential prefrontal and parietal responses to auditory and visual stimuli. Neuroimage 21:340-351.

Demerens C, Stankoff B, Logak M, Anglade P, Allinquant B, Couraud F, Zalc B, Lubetzki C (1996) Induction of myelination in the central nervous system by electrical activity. Proc Natl Acad Sci U S A 93:9887-9892.

Draganski B, Gaser C, Kempermann G, Kuhn HG, Winkler J, Büchel C, May A (2006) Temporal and spatial dynamics of brain structure changes during extensive learning. J Neurosci 26:6314-6317.

Friston KJ, Holmes A, Poline JB, Price CJ, Frith CD (1996) Detecting activations in PET and fMRI: levels of inference and power. Neuroimage 4:223-235. 
Gaser C (2006) VBM Toolbox. Retrieved December 1, 2009 from http://dbm.neuro.uni-jena.de/vbm/.

Genovese CR, Lazar NA, Nichols T (2002) Thresholding of statistical maps in functional neuroimaging using the false discovery rate. Neuroimage 15:870-878.

Goldman-Rakic PS (1988) Topography of cognition: parallel distributed networks in primate association cortex. Annu Rev Neurosci 11:137-156.

Goldman-Rakic PS (1994) Working memory dysfunction in schizophrenia. J Neuropsychiatry Clin Neurosci 6:348-357.

Good CD, Johnsrude I, Ashburner J, Henson RNA, Friston KJ, Frackowiak RSJ (2001) Cerebral asymmetry and the effects of sex and handedness on brain structure: a voxel-based morphometric analysis of 465 normal adult human brains. Neuroimage 14:685-700.

Hayasaka S, Nichols TE (2003) Validating cluster size inference: random field and permutation methods. Neuroimage 20:2343-2356.

Hsu YY, Huang CM, Kuan WC, Chen HM, Wai YY, Wan YL, Liu HL (2007) Spatial normalization of fMRI results using study-based EPI and T1weighted brain templates. Proc Intl Soc Magn Reson Med 15:1860.

Jaeggi SM, Buschkuehl M, Jonides J, Perrig WJ (2008) Improving fluid intelligence with training on working memory. Proc Natl Acad Sci U S A 105:6829-6833.

Jäncke L, Koeneke S, Hoppe A, Rominger C, Hänggi J (2009) The architecture of the golfer's brain. PLoS ONE 4:e4785.

Jones DK, Horsfield MA, Simmons A (1999) Optimal strategies for measuring diffusion in anisotropic systems by magnetic resonance imaging. Magn Reson Med 42:515-525.

Jones DK, Williams SCR, Gasston D, Horsfield MA, Simmons A, Howard R (2002) Isotropic resolution diffusion tensor imaging with whole brain acquisition in a clinically acceptable time. Hum Brain Mapp 15:216-230.

Jones DK, Symms MR, Cercignani M, Howard RJ (2005) The effect of filter size on VBM analyses of DT-MRI data. Neuroimage 26:546-554.

Juraska JM, Kopcik JR (1988) Sex and environmental influences on the size and ultrastructure of the rat corpus callosum. Brain Res 450:1-8.

Kanaan RAA, Kim JS, Kaufmann WE, Pearlson GD, Barker GJ, McGuire PK (2005) Diffusion tensor imaging in schizophrenia. Biological Psychiatry 58:921-929.

Kawashima R, Okita K, Yamazaki R, Tajima N, Yoshida H, Taira M, Iwata K, Sasaki T, Maeyama K, Usui N, Sugimoto K (2005) Reading aloud and arithmetic calculation improve frontal function of people with dementia. J Gerontol A Biol Sci Med Sci 60:380-384.

Klingberg T (2006) Development of a superior frontal-intraparietal network for visuo-spatial working memory. Neuropsychologia 44:21712177.

Klingberg T, Forssberg H, Westerberg H (2002) Increased brain activity in frontal and parietal cortex underlies the development of visuospatial working memory capacity during childhood. J Cogn Neurosci 14:1-10.

Koenig HL, Schumacher M, Ferzaz B, Thi AN, Ressouches A, Guennoun R, Jung-Testas I, Robel P, Akwa Y, Baulieu EE (1995) Progesterone synthesis and myelin formation by Schwann cells. Science 268:1500-1503.

Koester J (1985) Functional consequences of passive membrane properties of the neuron. In: Principles of neural science (Kandell ER, Schwartz JH, eds), pp 66-74. Amsterdam: Elsevier.

Le Bihan D (2003) Looking into the functional architecture of the brain with diffusion MRI. Nat Rev Neurosci 4:469-480.

Markham JA, Greenough WT (2004) Experience-driven brain plasticity: beyond the synapse. Neuron Glia Biol 1:351-363.
McDonald WI, Sears TA (1970) The effects of experimental demyelination on conduction in the central nervous system. Brain 93:583-598.

Moseley M (2002) Diffusion tensor imaging and aging-a review. NMR in Biomedicine 15:553-560.

Nuñez JL, Nelson J, Pych JC, Kim JHY, Juraska JM (2000) Myelination in the splenium of the corpus callosum in adult male and female rats. Brain Res Dev Brain Res 120:87-90.

Oldfield RC (1971) The assessment and analysis of handedness: the Edinburgh inventory. Neuropsychologia 9:97-113.

Olesen PJ, Nagy Z, Westerberg H, Klingberg T (2003) Combined analysis of DTI and fMRI data reveals a joint maturation of white and grey matter in a fronto-parietal network. Brain Res Cogn Brain Res 18:48-57.

Olesen PJ, Westerberg H, Klingberg T (2004) Increased prefrontal and parietal activity after training of working memory. Nat Neurosci 7:75-79.

Perrig WJ, Hollenstein M, Oelhafen S (2009) Can we improve fluid intelligence with training on working memory in persons with intellectual disabilities? J Cogn Educ Psychol 8:148-164.

Peters A (2002) The effects of normal aging on myelin and nerve fibers: a review. J Neurocytol 31:581-593.

Pfefferbaum A, Sullivan EV (2003) Increased brain white matter diffusivity in normal adult aging: relationship to anisotropy and partial voluming. Magn Reson Med 49:953-961.

Poline JB, Worsley KJ, Evans AC, Friston KJ (1997) Combining spatial extent and peak intensity to test for activations in functional imaging. Neuroimage 5:83-96.

Salat DH, Tuch DS, Greve DN, van der Kouwe AJW, Hevelone ND, Zaleta AK, Rosen BR, Fischl B, Corkin S, Rosas HD, Dale AM (2005) Agerelated alterations in white matter microstructure measured by diffusion tensor imaging. Neurobiol Aging 26:1215-1227.

Selemon LD, Goldman-Rakic PS (1988) Common cortical and subcortical targets of the dorsolateral prefrontal and posterior parietal cortices in the rhesus monkey: evidence for a distributed neural network subserving spatially guided behavior. J Neurosci 8:4049-4068.

Shaw P, Greenstein D, Lerch J, Clasen L, Lenroot R, Gogtay N, Evans A, Rapoport J, Giedd J (2006) Intellectual ability and cortical development in children and adolescents. Nature 440:676-679.

Shen S, Szameitat AJ, Sterr A (2007) VBM lesion detection depends on the normalization template: a study using simulated atrophy. Magn Reson Imaging 25:1385-1396.

Shrager P (1993) Axonal coding of action potentials in demyelinated nerve fibers. Brain Res 619:278-290.

Swadlow HA (1985) Physiological properties of individual cerebral axons studied in vivo for as long as one year. J Neurophysiol 54:1346-1362.

Uchida S, Kawashima R (2008) Reading and solving arithmetic problems improves cognitive functions of normal aged people: a randomized controlled study. Age 30:21-29.

Wager TD, Smith EE (2003) Neuroimaging studies of working memory: a meta-analysis. Cogn Affect Behav Neurosci 3:255-274.

Waxman SG (1980) Determinants of conduction velocity in myelinated nerve fibers. Muscle Nerve 3:141-150.

Wingfield A, Stine EAL, Lahar CJ, Aberdeen JS (1988) Does the capacity of working memory change with age? Exp Aging Res 14:103-107.

Yakovlev PI, Lecours AR (1967) The myelogenetic cycles of regional maturation of the brain. In: Regional development of the brain in early life (Minkowski A, ed), pp 3-70. Philadelphia: Davis. 